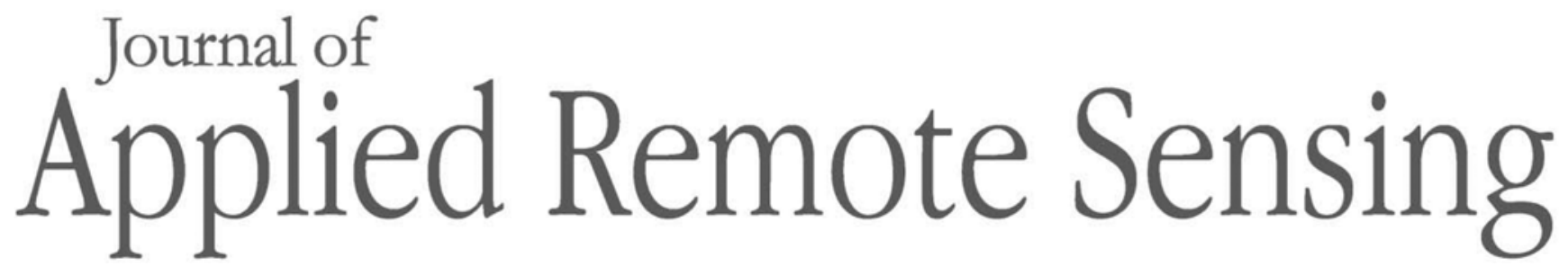

RemoteSensing.SPIEDigitalLibrary.org

\title{
Assessment of cross-sensor vegetation index compatibility between VIIRS and MODIS using near-coincident observations
}

Tomoaki Miura

Jordan Muratsuchi

Marco Vargas 


\title{
Assessment of cross-sensor vegetation index compatibility between VIIRS and MODIS using near-coincident observations
}

\author{
Tomoaki Miura, ${ }^{a}, *$ Jordan Muratsuchi, ${ }^{a}$ and Marco $\operatorname{Vargas}^{\mathrm{b}, \dagger}$ \\ ${ }^{a}$ University of Hawaii at Manoa, Department of Natural Resources and \\ Environmental Management, Honolulu, Hawaii, United States \\ ${ }^{b}$ National Oceanic and Atmospheric Administration, Office of Projects, \\ Planning, and Analysis, Silver Spring, Maryland, United States
}

\begin{abstract}
Cross-sensor compatibility of spectral vegetation indices (VIs) between Suomi National Polar-orbiting Partnership Visible Infrared Imaging Radiometer Suite (VIIRS) and Aqua Moderate Resolution Imaging Spectroradiometer (MODIS) was investigated using their near-coincident observation pairs obtained along overlapped orbital tracks across the globe for the year 2015. The "top-of-atmosphere (TOA)" and "top-of-canopy (TOC)" normalized difference vegetation indices (NDVIs), TOC-enhanced vegetation index (EVI), and TOC two-band EVI (EVI2) were investigated. For all four VIs, VIIRS and MODIS VIs were subject to systematic differences in which VIIRS VIs were higher than their MODIS counterparts. The overall systematic differences and uncertainties (measured as mean differences and root mean square differences, respectively) were small ( 0.010 to 0.020 VI units and 0.015 to 0.022 VI units, respectively). TOA NDVI cross-sensor differences were neither seasonally nor view zenith angle dependent, whereas TOC NDVI cross-sensor differences slightly varied seasonally, but were not view zenith angle dependent. TOC EVI and TOC EVI2 cross-sensor differences were view zenith angle dependent, where systematic differences increased with increasing view zenith angle and, for large view zenith angles, they were higher during the summer seasons. These results support the normalization of view zenith angles as a required step to extend the MODIS VI record with VIIRS data. (C) The Authors. Published by SPIE under a Creative Commons Attribution 3.0 Unported License. Distribution or reproduction of this work in whole or in part requires full attribution of the original publication, including its DOI. [DOI: 10.1117/1.JRS.12.045004]
\end{abstract}

Keywords: Visible Infrared Imaging Radiometer Suite; Moderate Resolution Imaging Spectroradiometer; normalized difference vegetation index; enhanced vegetation index; twoband enhanced vegetation index; data continuity.

Paper 180550 received Jul. 2, 2018; accepted for publication Sep. 14, 2018; published online Oct. 10, 2018.

\section{Introduction}

Spectral vegetation indices (VIs) have been used in a broad range of studies that involve an analysis of vegetation dynamics in regional-to-global scales, including climate-vegetation interactions, drought impact assessment, and land surface phenology. ${ }^{1-3}$ VIs have also been used successfully to estimate biophysical parameters, such as the fraction of photosynthetically active radiation, green vegetation fraction, leaf area index (LAI), and gross primary productivity (GPP). ${ }^{4-6}$ The most widely used index is the normalized difference vegetation index (NDVI): ${ }^{7,8}$

$$
\mathrm{NDVI}=\left(\rho_{\mathrm{NIR}}-\rho_{\mathrm{red}}\right) /\left(\rho_{\mathrm{NIR}}+\rho_{\mathrm{red}}\right),
$$

where $\rho_{\mathrm{NIR}}$ and $\rho_{\text {red }}$ are the near-infrared (NIR) and red reflectances, respectively. Long-term time-series data of the NDVI derived from the National Oceanic and Atmospheric

\footnotetext{
*Address all correspondence to: Tomoaki Miura, E-mail: tomoakim@ @awaii.edu

"This work was performed while the fourth author was at National Oceanic and Atmospheric Administration, Center for Satellite Applications and Research, College Park, Maryland, United States
} 
Administration (NOAA) Advanced Very High-Resolution Radiometer (AVHRR) sensor series (e.g., Ref. 9) have been used extensively to improve our understanding of climate-vegetation interactions in regional-to-global scales. ${ }^{1,10}$

Developed for the National Aeronautics and Space Administration (NASA) Moderate Resolution Imaging Spectroradiometer (MODIS) mission was the enhanced vegetation index (EVI) ${ }^{11}$ The EVI was designed to reduce the aerosol contaminations and canopy background brightness variations that were known to affect the NDVI, with improved sensitivity in high biomass regions where the NDVI tends to saturate: ${ }^{11}$

$$
\mathrm{EVI}=G \cdot\left(\rho_{\mathrm{NIR}}-\rho_{\text {red }}\right) /\left(\rho_{\mathrm{NIR}}+C_{1} \cdot \rho_{\text {red }}-C_{2} \cdot \rho_{\text {blue }}+L\right),
$$

where the blue reflectance ( $\rho_{\text {blue }}$ ) along with the two coefficients of $C_{1}$ and $C_{2}$ were introduced to reduce the aerosol influences and the $L$ factor was introduced to reduce the canopy background brightness effects. $G=2.5, C_{1}=6.0, C_{2}=7.5$, and $L=1.0$ were adopted for MODIS. The EVI was instrumental in examining how climate controls vegetation photosynthetic activities in tropical forests (e.g., Ref. 12). Recently, Seddon et al. ${ }^{13}$ developed a quantitative methodology for assessing the relative sensitivity of terrestrial ecosystems to climate variability using the MODIS EVI time-series data on a global scale.

Although many studies found that the EVI was advantageous in vegetation productivity assessments, ${ }^{14}$ the EVI is applicable only to those sensor systems with a blue band in addition to red and NIR bands by its very design. Likewise, the EVI can result in faulty values for snow-/ice-contaminated observations that have higher blue and red reflectances than NIR reflectance. ${ }^{11,15}$ A two-band version of the EVI without a blue band, or EVI2, was introduced by Jiang et al. ${ }^{16}$ to overcome these issues:

$$
\mathrm{EVI} 2=2.5 \cdot\left(\rho_{\mathrm{NIR}}-\rho_{\mathrm{red}}\right) /\left(\rho_{\mathrm{NIR}}+2.4 \cdot \rho_{\mathrm{red}}+1.0\right) .
$$

The three coefficients in the EVI2 equation were optimized to attain the best similarity with the EVI for the MODIS bandpasses and, thus, the EVI2 can be used as an exact substitute of the EVI for good observations. ${ }^{16}$ Didan et al. ${ }^{17}$ developed a multisensor EVI2 time-series dataset from AVHRR and MODIS. This dataset was used to assess the effect of early spring warming on vegetation growth in the conterminous United States, ${ }^{18}$ and interannual variations and trends in global land surface phenology. ${ }^{19}$ The EVI2 has also been applied to other sensors, even those with a blue band (e.g., Landsat). ${ }^{20}$

Visible Infrared Imaging Radiometer Suite (VIIRS) is a new polar-orbiting satellite sensor series that replaces NOAA AVHRR with afternoon overpass and is slated to continue the highly calibrated data stream initiated with NASA MODIS. ${ }^{21,22}$ The first VIIRS sensor, on board the Suomi National Polar-orbiting Partnership (S-NPP), was launched in November 2011. Since 2012, a number of standard geophysical products, referred to as Environmental Data Records (EDRs), have been produced from VIIRS-measured radiance data. VIIRS vegetation index EDR is a 375-m (at nadir) daily global product and includes the "top-of-atmosphere (TOA)" NDVI as the continuity index from AVHRR and the atmospherically corrected "topof-canopy (TOC)" EVI as the MODIS heritage index. ${ }^{15}$ The second VIIRS sensor was launched on the Joint Polar Satellite System 1 (JPSS-1) platform in November 2017, which is expected to continue the current suite of VIIRS EDRs.

Although VIIRS data products are expected to continue and complement the MODIS counterparts, VIIRS and MODIS differ slightly in their sensor and platform characteristics (Table 1), which can cause systematic differences between their VI time-series data. Several studies investigated VIIRS versus MODIS cross-sensor VI compatibility by band simulation analyses with Earth Observing-One (EO-1) Hyperion hyperspectral imagery ${ }^{23-25}$ or by cross comparisons of actual VIIRS and MODIS data. ${ }^{15,26,27}$ For the NDVI, Vargas et al. ${ }^{15}$ compared VIIRS and MODIS TOA NDVI using their near-coincident, near-nadir (view zenith angle $<7.5 \mathrm{deg}$ ) observation pairs obtained over the western hemisphere for May 2012 to March 2013 and found that VIIRS TOA NDVI was higher than the MODIS counterpart with the mean difference (MD) of $\sim 0.02$ NDVI units. Skakun et al. ${ }^{27}$ also used VIIRS and MODIS near-coincident, near-nadir observation pairs, but extracted over the central US region for a period from 2012 to 2016, 
Table 1 S-NPP VIIRS and Aqua MODIS sensor and platform characteristics relevant to vegetation index products (adapted from Vargas et al. $^{15}$ ).

\begin{tabular}{|c|c|c|}
\hline & S-NPP VIIRS & Aqua MODIS \\
\hline Altitude & 833 km & 705 km \\
\hline Orbit & Near-polar, sun-synchronous & Near-polar, sun-synchronous \\
\hline Equator crossing time & 1:30 pm (ascending) & 1:30 pm (ascending) \\
\hline Repeat cycle & 16 days & 16 days \\
\hline Orbital cycle & 14 times per day & 16 times per day \\
\hline Swath width & $\begin{array}{l}112 \text { deg }( \pm 56 \mathrm{deg}), 3000 \mathrm{~km} \\
\text { (whiskbroom) }\end{array}$ & $\begin{array}{l}110 \text { deg }( \pm 55 \text { deg), } 2330 \mathrm{~km} \\
\text { (whiskbroom) }\end{array}$ \\
\hline \multirow[t]{3}{*}{ Spectral bands $(\mathrm{nm})$} & $\operatorname{Red}($ (I1): 640 (600 to 680$)$ & Red: 646 (620 to 670$)$ \\
\hline & NIR (I2): 865 (850 to 880 ) & NIR: 857 (841 to 876$)$ \\
\hline & Blue (M3): 488 (478 to 488 ) & Blue: 466 (459 to 479 ) \\
\hline \multirow[t]{8}{*}{ Spatial resolution } & Red (I1) and NIR (I2) & Red and NIR \\
\hline & $375 \mathrm{~m}$ at nadir & $250 \mathrm{~m}$ at nadir \\
\hline & $0.55 \times 0.62 \mathrm{~km}$ at $\theta_{v}=50 \mathrm{deg}^{\mathrm{a}}$ & $0.4 \times 0.7 \mathrm{~km}$ at $\theta_{v}=55 \mathrm{deg}$ \\
\hline & $0.8 \times 0.8 \mathrm{~km}$ at edge $\left(\theta_{v}=69.5 \mathrm{deg}\right)$ & $0.5 \times 1.2 \mathrm{~km}$ at edge $\left(\theta_{v}=65.4 \mathrm{deg}\right)$ \\
\hline & Blue (M3) & Blue \\
\hline & $750 \mathrm{~m}$ at nadir & $500 \mathrm{~m}$ at nadir \\
\hline & $1.1 \times 1.26 \mathrm{~km}$ at $\theta_{v}=50 \mathrm{deg}$ & $0.8 \times 1.4 \mathrm{~km}$ at $\theta_{v}=55 \mathrm{deg}$ \\
\hline & $1.6 \times 1.6 \mathrm{~km}$ at edge $\left(\theta_{v}=69.5 \mathrm{deg}\right)$ & $1 \times 2.4 \mathrm{~km}$ at edge $\left(\theta_{v}=65.4 \mathrm{deg}\right)$ \\
\hline
\end{tabular}

${ }^{\mathrm{a}} \theta_{v}-$ Satellite view zenith angle.

and showed that VIIRS TOC NDVI was higher than the MODIS counterpart (the MD of 0.021 NDVI units).

For the EVI, Hyperion band simulation analyses over a tropical forest-savanna ecogradient in Brazil $^{23}$ and over AERONET sites across the globe ${ }^{25}$ both showed that VIIRS EVI was higher than MODIS EVI. In their analysis with the same near-coincident, near-nadir observation pairs as used in the TOA-NDVI analysis, Vargas et al. ${ }^{15}$ showed that the mean of VIIRS EVI minus MODIS EVI was zero when EVI was zero, but always positive for the rest of the EVI dynamic range. Another EVI compatibility analysis using a global S-NPP VIIRS and Aqua MODIS dataset from the year 2013 reported that the MD of VIIRS EVI minus MODIS EVI was 0.021 EVI units. ${ }^{26}$ For the EVI2, Miura et al. ${ }^{25}$ indicated, in their Hyperion band simulation analysis, that VIIRS EVI2 could be higher or lower than MODIS EVI2, depending on the accuracy of aerosol corrections. Zhang et al. ${ }^{28}$ cross-compared EVI2 time-series datasets derived from S-NPP VIIRS and Terra MODIS $5 \mathrm{~km}$ (climate modeling grid, CMG) daily global surface reflectance products. Their results indicated that VIIRS EVI2 was randomly higher or lower than MODIS EVI2, while their time series was similar.

Several studies applied spectral adjustments to VIIRS data and evaluated the improvements in VIIRS-to-MODIS cross-sensor VI consistency/compatibility using actual VIIRS and MODIS data. Skakun et al. ${ }^{27}$ weight-averaged VIIRS red and NIR bands (with different weights) to spectrally adjust VIIRS red and NIR reflectances to their MODIS counterparts and obtained a MODIS-equivalent VIIRS NDVI with the adjusted VIIRS reflectances. Obata et al. ${ }^{26}$ crosscalibrated VIIRS EVI-to-MODIS EVI and obtained a MODIS-compatible VIIRS EVI with a year-long, global VIIRS-MODIS CMG dataset. The calibrated MODIS-compatible VIIRS 
EVI showed much higher compatibility with the MODIS EVI, where the MD (VIIRS minus MODIS) decreased from 0.021 to 0.003 EVI units.

In this study, we extend the approach of Vargas et al. ${ }^{15}$ and cross-compare S-NPP VIIRS and Aqua MODIS VIs using their near-coincident observation pairs collected for a wide range of view zenith angles across the globe for the year 2015. VIIRS-MODIS cross-sensor VI differences were examined to assess their radiometric compatibility across view zenith angles, as previous studies were limited to comparing near-nadir observations or temporally composited time-series data. In addition to TOA NDVI and TOC EVI, TOC NDVI and TOC EVI2 were investigated. As VIIRS and MODIS spectral bandpasses are slightly different, their corresponding spectral reflectances should have slightly different sun-target-view angle dependencies, or bidirectional reflectance distribution functions (BRDFs), which would propagate into their cross-sensor VI differences.

\section{Materials}

S-NPP VIIRS and Aqua MODIS sensor and platform characteristics relevant to VI products are shown in Table 1. The VIRS red band is wider than the MODIS counterpart, whereas the VIIRS NIR band is positioned at slightly longer wavelengths than the MODIS counterpart (Table 1). The VIIRS and MODIS blue bandpasses barely overlap where the former encompasses longer wavelengths than the latter. In addition to the spectral bandpass differences described in the previous section, they differ in spatial resolution and platform orbit. VIIRS provides lower spatial resolution data than MODIS (Table 1). However, VIIRS uses a unique pixel aggregation scheme that controls the pixel growth toward the scan edge. ${ }^{29}$ The VIIRS pixel size only doubles at the edge of scan.

Although the two satellites have a 16-day repeat cycle, S-NPP orbits 14 times per day and Aqua orbits 16 times per day. VIIRS and MODIS orbital tracks have considerable overlaps over land on 3 days over every 8-day period (a half of their 16-day repeat cycle). For example, they overlapped on days of year (DOYs) 27, 29, and 32 for an 8-day period starting on 25 January (DOY 25) 2015. The overlapped tracks on each of the 3 days cover different parts of the Earth but provides near-global coverage when combined (Fig. 1).

VIIRS and MODIS products were obtained for those 3 days at monthly (32-day) intervals for the year 2015 (Table 2). For VIIRS, Archive Set (AS) 3001 VI EDR, daily TOA and surface reflectances, and geoangle products were obtained from the Level-1 and Atmosphere Archive and Distribution System (LAADS) Distributed Active Archive Center (DAAC) ${ }^{30}$ VIIRS algorithms for all these products had reached a "validated" status by January $2015,{ }^{31}$ in which the actual products met their respective accuracy and precision requirements defined by the JPSS

(a)

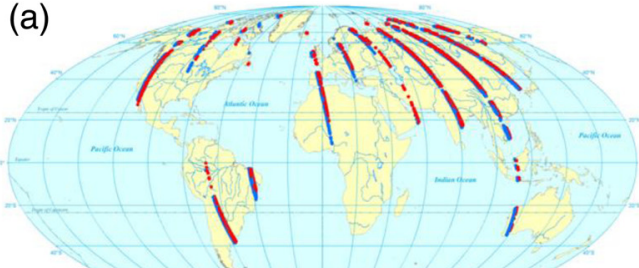

(c)

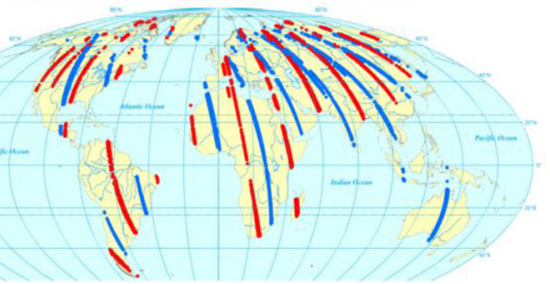

(b)

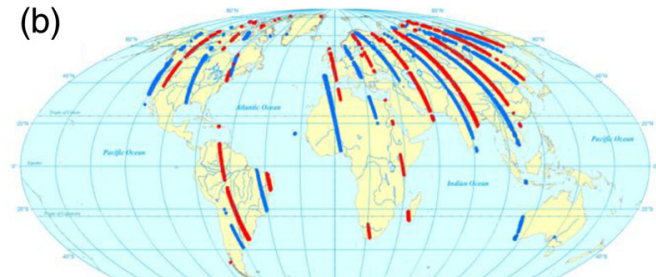

(d)

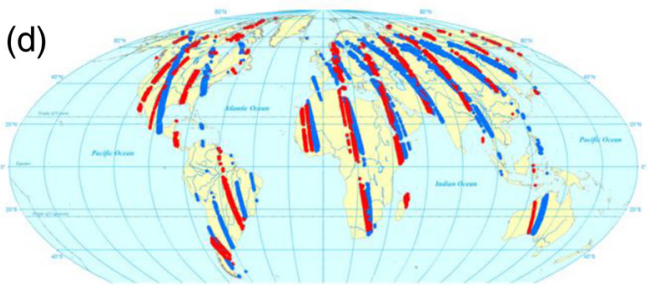

Fig. 1 Geographic distribution of S-NPP VIIRS and Aqua MODIS observation pairs used in this study. The red and blue colors are for the backward and forward observation geometries, respectively: (a) $0 \mathrm{deg} \leq \theta_{v}<7.5 \mathrm{deg}$, (b) $20 \mathrm{deg} \leq \theta_{v}<27.5 \mathrm{deg}$, (c) $40 \mathrm{deg} \leq \theta_{v}<47.5 \mathrm{deg}$, and (d) $55 \mathrm{deg} \leq \theta_{v}<62.5 \mathrm{deg}$. 
Miura, Muratsuchi, and Vargas: Assessment of cross-sensor vegetation index compatibility...

Table 2 VIIRS and MODIS data days used in this study.

\begin{tabular}{lccccccccccc}
\hline \hline & Jan to Feb & Mar & Apr & May & Jun & Jul & Aug & Sep & Oct & Nov & Dec \\
\hline Day of year & 027 & 059 & 091 & 123 & 155 & 187 & 219 & 251 & 283 & 315 & 347 \\
& 029 & 061 & 093 & 125 & 157 & 189 & 221 & 253 & 285 & 317 & 349 \\
& 032 & 064 & 096 & 128 & 160 & 192 & 224 & 256 & 288 & 320 & 352 \\
\hline \hline
\end{tabular}

program..$^{32}$ For MODIS, Collection 6 daily 500-m TOA and surface reflectance (MYD02HKM and MYD09, respectively) and geoangle (MYD03) products were obtained from the LAADS DAAC. These MODIS products had achieved a "validated stage 3" status. ${ }^{33}$

As all of the above VIIRS and MODIS products were unprojected granules, they were first remapped onto a geographic projection grid with nearest neighbor resampling and stitched into daily global mosaics. The grid sizes of $0.0036 \mathrm{deg} \times 0.004 \mathrm{deg}$ (north-south and east-west directions, respectively) and $0.004 \mathrm{deg} \times 0.004 \mathrm{deg}$ were adopted for VIIRS and MODIS, respectively.

The global mosaic data were then screened for quality and sun-target-view geometries. Perpixel quality assurance (QA) information contained in VIIRS VI EDR and MODIS surface reflectance products were used to retain high-quality observations. Following the high-quality definition used in Vargas et al., ${ }^{15}$ pixels with any of the following QA flags were removed: not confidently clear, adjacent to cloud, cloud shadow, snow or ice, thin cirrus cloud, high aerosol loading, solar zenith angle $>65 \mathrm{deg}$, and not over land. Pixels under the following eight view zenith $\left(\theta_{v}\right)$ and relative azimuth $\left(\phi_{r}\right)$ angle bins were retained, which covered a range of view zenith angles encountered by VIIRS and MODIS (see Table 1): $0 \mathrm{deg} \leq \theta_{v}<7.5 \mathrm{deg}$, $20 \mathrm{deg} \leq \theta_{v}<27.5 \mathrm{deg}, 40 \mathrm{deg} \leq \theta_{v}<47.5 \mathrm{deg}$, and $55 \mathrm{deg} \leq \theta_{v}<62.5 \mathrm{deg}$ in the forward (90 deg $\left.<\phi_{r} \leq 180 \mathrm{deg}\right)$ and backward ( $\left.0 \mathrm{deg} \leq \phi_{r} \leq 90 \mathrm{deg}\right)$ scattering directions, respectively. VIIRS TOC NDVI and TOC EVI2, which were not part of the standard VIIRS VI EDR, and MODIS TOA NDVI, TOC NDVI, TOC EVI, and TOC EVI2 were computed from their respective reflectances after the screening.

Finally, the global mosaic data were spatially aggregated into a coarser grid ( $0.036 \mathrm{deg} \times 0.04 \mathrm{deg}$ or $\sim 4 \mathrm{~km})$. This grid size was selected to minimize the impact of misregistration between VIIRS and MODIS pixels and because it is the standard grid size used for the accuracy assessment of VIIRS VI EDR. ${ }^{32}$ Aggregated values were computed only when the number of pixels found in a coarser grid was $\geq 80$ pixels for VIIRS and 72 pixels for MODIS (i.e., $80 \%$ of the maximum number).

VIIRS and MODIS observation pairs belonging to the same view zenith-relative azimuth angle bins, or geoangle bins were extracted from the 4-km global mosaics. For each of the eight geoangle bins, the extracted observation pairs from a set of three days were combined to form a single, monthly dataset. A subsample of size 2000 was selected from this monthly dataset randomly, but to have uniform sampling frequencies across the VI dynamic range. ${ }^{34}$ We created one subsample dataset for the TOA NDVI and TOC NDVI, and another subsample dataset for the TOC EVI and TOC EVI2, as the NDVIs and the EVI/EVI2 had completely different histograms when the same subsample was used.

Resultant subsamples ranged in size from 1500 to 2500 across seasons and geoangle bins, with the mean size being 2360 for the NDVIs and 2034 for the EVI/EVI2, as the total number of available observation pairs seasonally and spatially varied. The subsample dataset all together had global coverage, but the sampled geographic areas slightly differed among geoangle bins, the limitation due to the overlapped orbit occurrences (Fig. 1). For example, nadir and near-nadir observation pairs had smaller coverage over the African continent [Figs. 1(a) and 1(b)] than off-nadir observation pairs [Figs. 1(c) and 1(d)].

Solar zenith angle differences of the subsample dataset ranged from 0 deg to 2 deg in the backward scattering direction and from 0 deg to $3.5 \mathrm{deg}$ in the forward scattering direction, where solar zenith angles themselves ranged from $10 \mathrm{deg}$ to $65 \mathrm{deg}$. Solar zenith angle differences of $>2$ deg occurred only when the view zenith angles of the corresponding observation pairs were $>40 \mathrm{deg}$. Relative azimuth angle differences were, on average, $0 \mathrm{deg}$ to $5 \mathrm{deg}$ 
Miura, Muratsuchi, and Vargas: Assessment of cross-sensor vegetation index compatibility...

Table 3 Reflectance and VI dynamic range (99.9\%).

\begin{tabular}{lccc}
\hline \hline & Dynamic range & 0.05th percentile & 99.95th percentile \\
\hline Red TOA reflectance & 0.44 & 0.03 & 0.47 \\
NIR TOA reflectance & 0.51 & 0.09 & 0.60 \\
Red TOC reflectance & 0.49 & 0.01 & 0.50 \\
NIR TOC reflectance & 0.52 & 0.09 & 0.61 \\
Blue TOC reflectance & 0.23 & 0.00 & 0.23 \\
TOA NDVI & 0.76 & 0.03 & 0.79 \\
TOC NDVI & 0.86 & 0.04 & 0.90 \\
TOC EVI & 0.79 & 0.03 & 0.82 \\
TOC EVI2 & 0.78 & 0.03 & 0.81 \\
\hline \hline
\end{tabular}

in the backward and $0 \mathrm{deg}$ to $12 \mathrm{deg}$ in the forward scattering direction, where relative azimuth angles themselves ranged from $0 \mathrm{deg}$ to $70 \mathrm{deg}$ and from $105 \mathrm{deg}$ to $180 \mathrm{deg}$ for the former and latter, respectively.

Dynamic ranges of the VIs and input reflectances were derived from the subsample dataset and are summarized in Table 3. To reduce the impact of potential outliers, the 0.05 th and 99.95th percentiles were determined for VIIRS and MODIS. The means of the VIIRS and MODIS percentiles were used to define the dynamic ranges. It should be noted that TOC reflectances and TOC NDVI had a wider dynamic range than their TOA counterparts due to atmospheric correction and that TOC EVI and TOC EVI2 had nearly the same dynamic ranges, an indication of their general compatibility.

\section{Analysis Methods}

We first investigated cross-sensor reflectance and VI relationships by plotting their cross-sensor differences (VIIRS minus MODIS) against MODIS values for each geoangle bin and for each month. Second, three difference statistics were computed per geoangle bin and per month, and their trends with respect to view zenith angles and seasons were examined for each of the four VIs. They were MDs, standard deviations of the difference (SDs), and root mean square differences (RMSDs) and used as a measure of systematic difference, random variation, and uncertainty, respectively:

$$
\begin{gathered}
\mathrm{MD}=\frac{1}{n} \sum_{i=1}^{n}\left(x_{i, \mathrm{VIIRS}}-x_{i, \mathrm{MODIS}}\right), \\
\mathrm{SD}=\sqrt{\frac{1}{n-1} \sum_{i=1}^{n}\left[\left(x_{i, \mathrm{VIIRS}}-x_{i, \mathrm{MODIS}}\right)-\mathrm{MD}\right]^{2},} \\
\mathrm{RMSD}=\sqrt{\frac{1}{n} \sum_{i=1}^{n}\left(x_{i, \mathrm{VIIRS}}-x_{i, \mathrm{MODIS}}\right)^{2},}
\end{gathered}
$$

where $x_{i, \mathrm{VIIRS}}$ and $x_{i, \mathrm{MODIS}}$ are the reflectance or VI values of VIIRS and MODIS, respectively, for the 4-km grid cell $i$, and $n$ is the sample size (the number of pairs). These difference statistics were also converted into \% relative values (i.e., \% relative MD, SD, and RMSD) by dividing them by the $99.9 \%$ dynamic ranges of the corresponding variables, which allowed for comparisons of VIIRS and MODIS compatibilities across different VIs. 
Finally, to identify the spectral bands responsible for observed trends in VIIRS versus MODIS VI differences, we decomposed VI differences into their band components. The following error propagation equation was employed for this purpose $:^{35}$

$$
\Delta \mathrm{VI} \approx \frac{\partial \mathrm{VI}}{\partial \rho_{\mathrm{NIR}}} \cdot \Delta \rho_{\mathrm{NIR}}+\frac{\partial \mathrm{VI}}{\partial \rho_{\text {red }}} \cdot \Delta \rho_{\text {red }}+\frac{\partial \mathrm{VI}}{\partial \rho_{\text {blue }}} \cdot \Delta \rho_{\text {blue }}
$$

where $\Delta \mathrm{VI}, \Delta \rho_{\text {red }}, \Delta \rho_{\text {NIR }}$, and $\Delta \rho_{\text {blue }}$ are the VI, red, NIR, and blue reflectance differences, respectively, between VIIRS and MODIS (VIIRS minus MODIS). This error propagation [Eq. (7)] is based on a first-order Taylor series approximation of the VI equation and indicates that the cross-sensor VI difference consists of three band components, each of which is a product of the cross-sensor reflectance difference (e.g., $\Delta \rho_{\text {red }}$ ) and the partial derivative of the VI at the mean reflectances of the two sensors (e.g., $\frac{\partial \mathrm{VI}}{\partial \rho_{\mathrm{red}}}$ ) ${ }^{23}$ For the NDVI and EVI2, the third term in the right side of Eq. (7) is zero, as the partial derivative of the NDVI and EVI2 equations with respect to the blue reflectance is zero. The partial derivatives of the NDVI, EVI, and EVI2 are provided in the Appendix.

\section{Results}

\subsection{Compatibility over Dynamic Range}

In Fig. 2, VIIRS versus MODIS reflectance differences are plotted as a function of MODIS values for nadir-to-near-nadir viewing conditions (i.e., $0 \leq \theta_{v}<7.5$ for all relative azimuth angles) for August 2015. Reflectance difference statistics (MD and SD), which were computed on a per-bin basis, are also plotted to observe the trends and variations of the differences over the dynamic ranges. Red TOA reflectance differences were, on average, nearly zero at its low value range ( 0 to 0.15$)$ and then linearly decreased (increased negatively) with increasing reflectance, i.e., VIIRS red TOA reflectance was lower than the MODIS counterpart [Fig. 2(a)]. In general, NIR TOA differences were positive across its dynamic range and increased with increasing reflectance [Fig. 2(b)]. Red TOC reflectance differences showed a similar trend to red TOA reflectance differences, but the former's MD was negative throughout its dynamic range [Fig. 2(c)]. NIR TOC reflectance differences were, on average, also positive, but they were slightly smaller than NIR TOA reflectance differences [Fig. 2(d)]. Blue TOC reflectance differences were nearly zero when the reflectance was $<0.025$, but they were positive and increased with increasing reflectance values [Fig. 2(e)].

Reflectance differences and difference statistics for August 2015, but for off-nadir viewing in the backward scattering direction (55 deg $\leq \theta_{v}<62.5 \mathrm{deg}$ and $0 \mathrm{deg} \leq \phi_{r} \leq 90 \mathrm{deg}$ ), are plotted in Fig. 3. Cross-sensor reflectance differences for this off-nadir viewing condition were different from those observed for the near-nadir viewing condition. Red TOA reflectance differences were basically nearly zero throughout its dynamic range [Fig. 3(a)]. Red TOC reflectance differences were also nearly zero when reflectance values were $<0.3$ [Fig. 3(b)]. They negatively increased with increasing reflectances, but were smaller than those observed for the near-nadir viewing condition. NIR TOA and TOC reflectance differences for this off-nadir viewing condition were larger than those observed for the near-nadir viewing [Figs. 3(c) and 3(d)]. Blue TOC reflectance differences for this off-nadir viewing geometry were about the same as those for the near-nadir viewing geometry when its reflectance values were $<0.1$, but they were larger when the reflectance values were $>0.12$ [Fig. 3(e)].

In Fig. 4, VI differences are plotted as a function of MODIS VIs for nadir-to-near-nadir viewing conditions (i.e., $0 \leq \theta_{v}<7.5$ for all relative azimuth angles) for August 2015. To observe the trends and variations of the differences over the dynamic ranges, VI difference statistics (MD and SD), which were computed on a per-bin basis with each bin having the size of 0.025 VI units, are also plotted. For all four VIs examined, VI differences were generally positive and MDs were positive throughout the VI dynamic ranges, except for TOA NDVI when its values were $>0.6$. Thus, VIIRS VIs were higher than the MODIS counterparts in general. TOA NDVI MD was the highest $(\sim 3 \%)$ at the low NDVI values of 

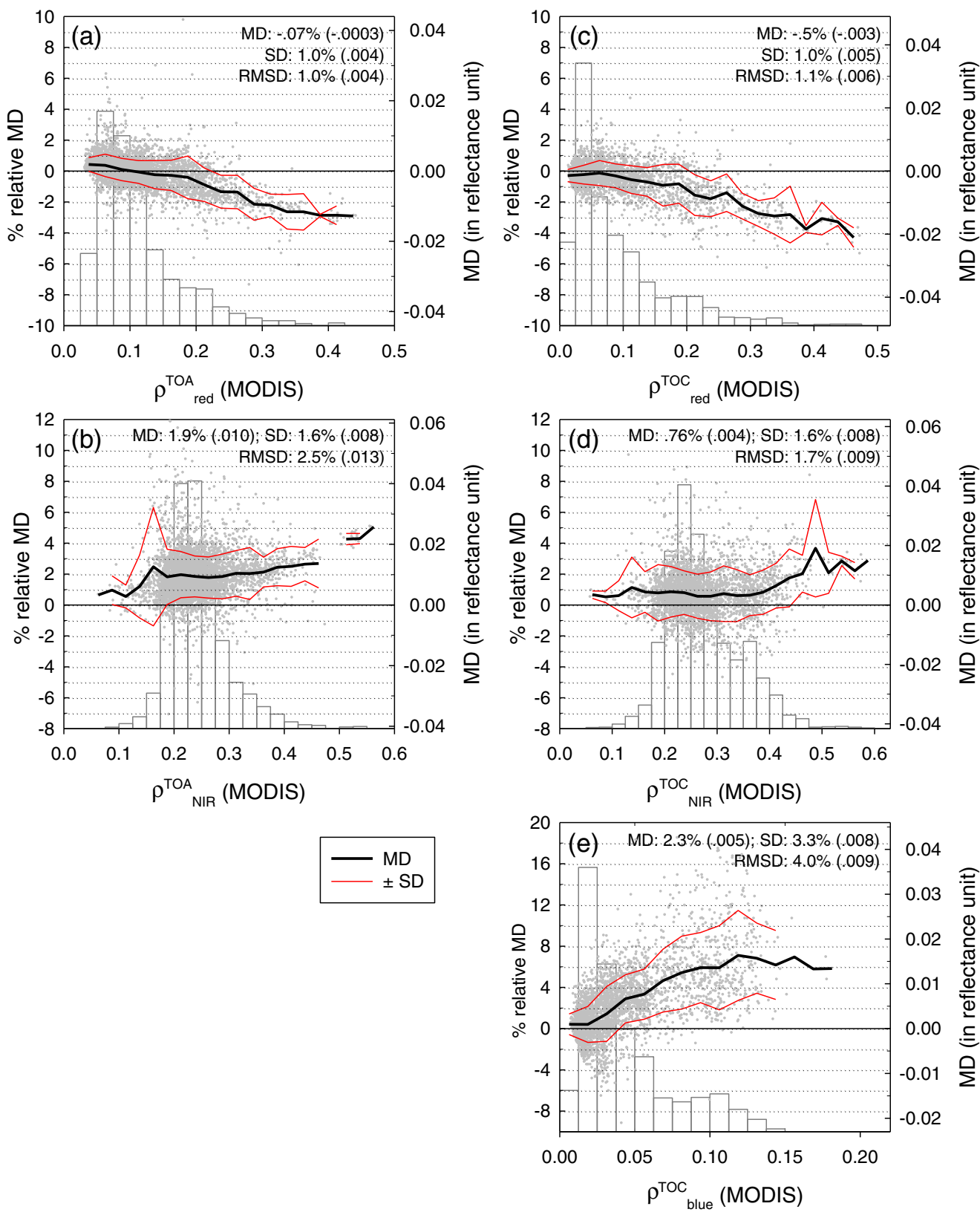

Fig. 2 VIIRS versus MODIS reflectance differences and difference statistics (VIIRS minus MODIS) plotted against MODIS values for nadir-to-near-nadir viewing condition ( 0 deg $\leq$ $\theta_{v}<7.5$ deg for all relative azimuth angles) for August 2015: (a) red TOA reflectance, (b) NIR TOA reflectance, (c) red TOC reflectance, (d) NIR TOC reflectance, and (e) blue TOC reflectance. The left scale is for percent relative difference and the right scale for absolute difference. Shown at the bottom of each plot is the histogram of observation pairs.

0.1 to 0.3 decreased with increasing NDVI values, and were nearly zero when NDVI $>0.6$ [Fig. 4(a)]. TOC NDVI MD was also higher $(\sim 2 \%)$ for low NDVI values $(<0.3)$ than for high NDVI values $(>0.5)$, but positive throughout its dynamic range [Fig. 4(b)]. TOC EVI MD was around $2.5 \%$ when EVI $<0.35$ and $\sim 1.5 \%$ when EVI $>0.35$ [Fig. 4(c)]. VIIRS TOC EVI2 was consistently higher than the MODIS counterpart with their MD being $1.3 \%$, on average [Fig. 4(d)].

Plotted in Fig. 5 are VI differences and difference statistics for August 2015, but for off-nadir viewing in the backward scattering direction (55 deg $\leq \theta_{v}<62.5 \mathrm{deg}$ and $0 \mathrm{deg} \leq$ $\left.\phi_{r} \leq 90 \mathrm{deg}\right)$. VI differences and MDs were also generally positive throughout VI dynamic 

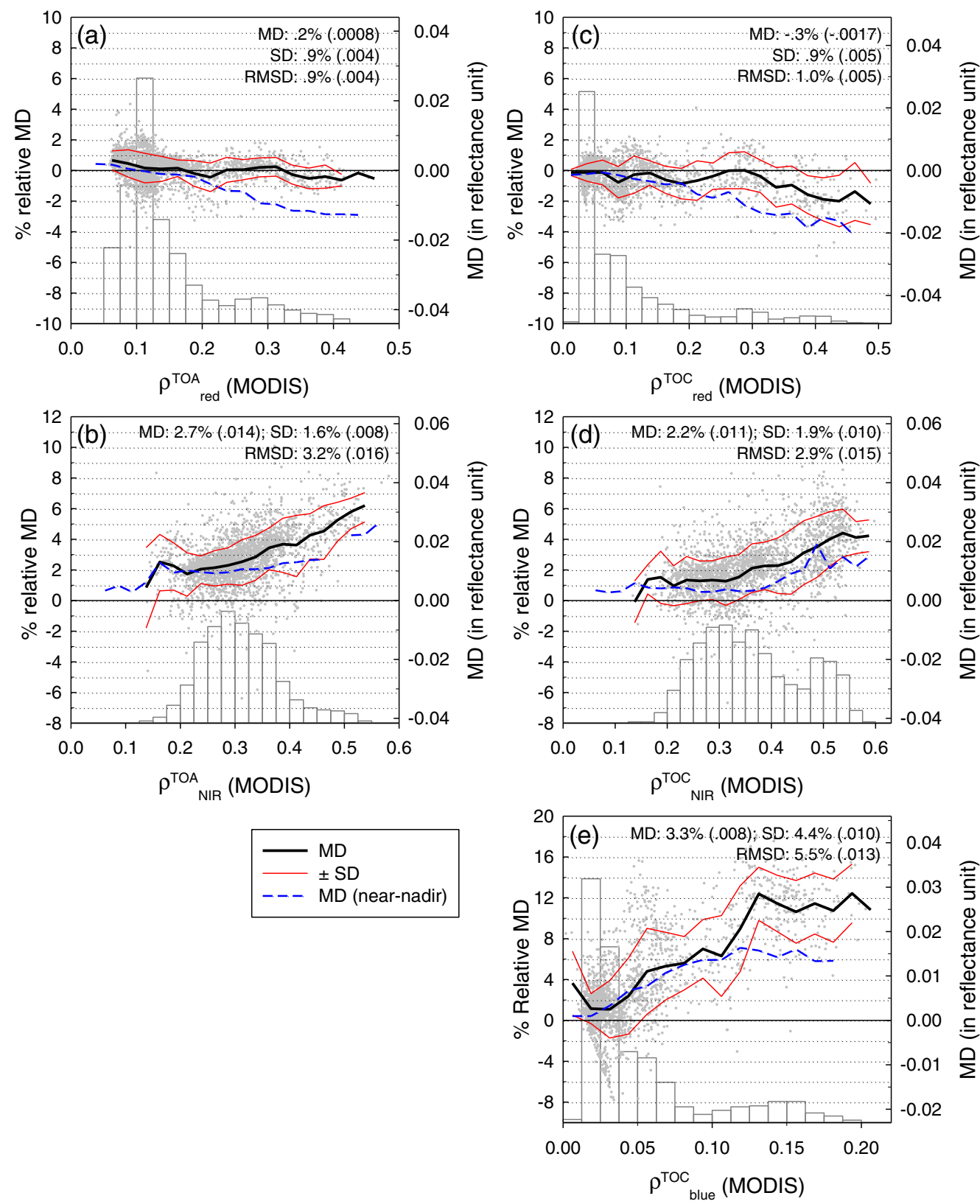

Fig. 3 Same as Fig. 2, but for off-nadir viewing in the backward scattering direction (55 deg $\leq \theta_{v}<62.5 \mathrm{deg}$ and $0 \mathrm{deg} \leq \phi_{r} \leq 90 \mathrm{deg}$ ).

ranges for all the four VIs, except for TOA NDVI when its values were $>0.6$. TOA NDVI MD for this off-nadir viewing condition, which was lower than that for the near-nadir viewing condition over the NDVI range of 0.15 to 0.4 [Fig. 5(a)], whereas TOC NDVI MD for this off-nadir viewing condition was slightly higher than that for the near-nadir condition over the 0.5 to 0.6 NDVI range [Fig. 5(b)]. However, both TOA NDVI and TOC NDVI MDs for the off-nadir viewing condition had very similar trends to those for the near-nadir viewing condition. In contrast, TOC EVI MD for the off-nadir viewing condition was consistently higher than that for the nearnadir viewing condition across its dynamic range, being $\sim 1.5$ times larger (i.e., $3.4 \%$ versus $2.2 \%$ ) on average [Fig. 5(c)]. TOC EVI2 MD for the off-nadir viewing condition was also higher than the near-nadir counterpart, but only for high EVI2 values (>0.5) [Fig. 5(d)]. Both TOC EVI and TOC EVI2 had a wider dynamic range for the off-nadir viewing condition than for the near-nadir viewing condition. 

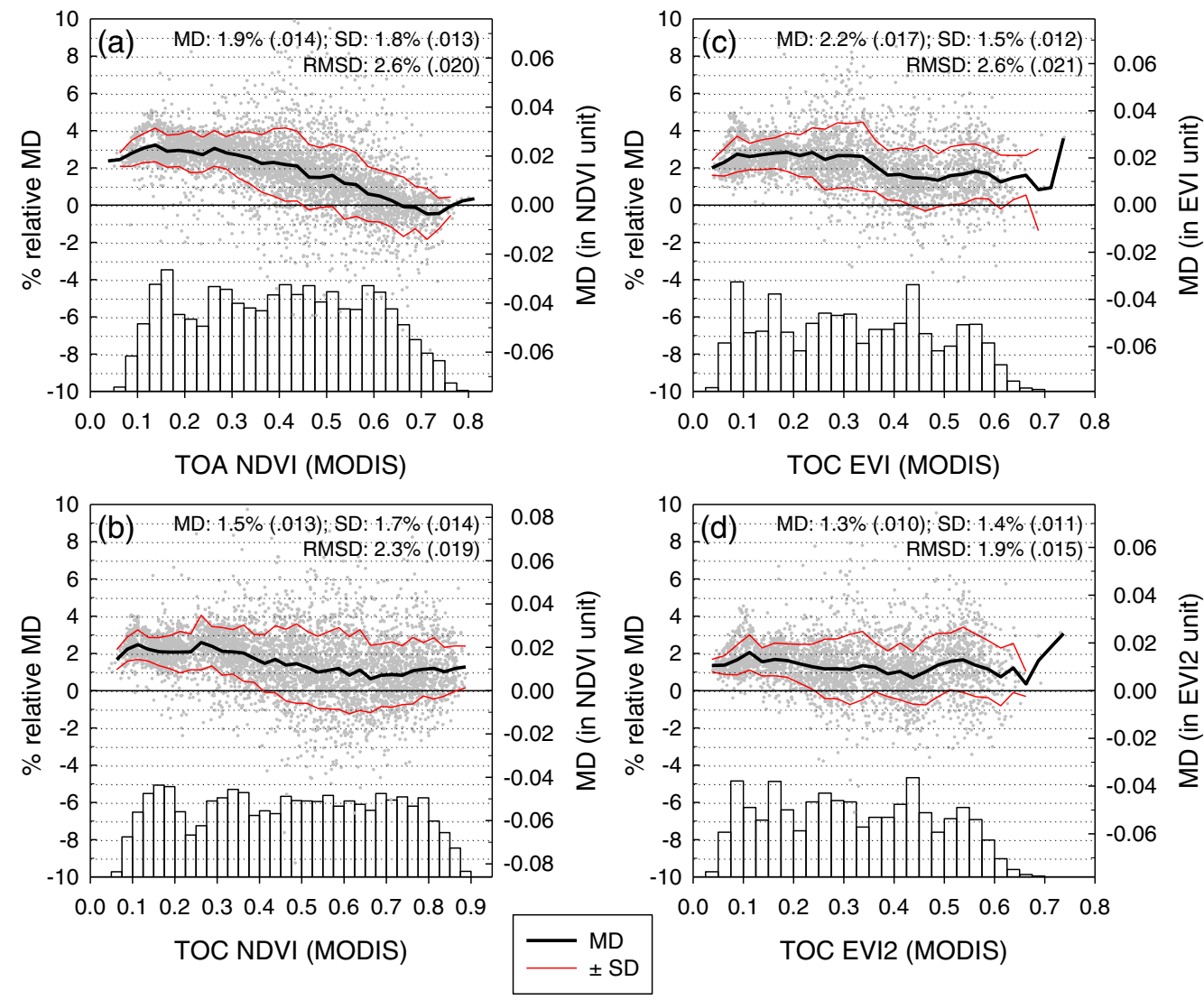

Fig. 4 VIIRS versus MODIS VI differences and difference statistics (VIIRS minus MODIS) plotted against MODIS VI values for nadir-to-near-nadir viewing condition ( $0 \mathrm{deg} \leq \theta_{v}<7.5 \mathrm{deg}$ for all relative azimuth angles) for August 2015: (a) TOA NDVI, (b) TOC NDVI, (c) TOC EVI, and (d) TOC EVI2. The left scale is for percent relative difference and the right scale for absolute difference. Shown at the bottom of each plot is the histogram of observation pairs. The bin size is $0.025 \mathrm{VI}$ units.

\subsection{Compatibility over View Zenith Angle}

In Fig. 6, VI difference statistics are plotted as a function of view zenith angle to examine VIIRSMODIS cross-sensor compatibility across a range of view zenith angles for three selected months that represented the trends observed for the other months. For TOA NDVI, MD, $\mathrm{SD}$, and RMSD remained nearly the same across view zenith angles for all of the 3 months [Fig. 6(a)]. For TOC NDVI, MD varied with view zenith angles, but its variations did not have any systematic trends with respect to view zenith angles [Fig. 6(b)]. TOC NDVI SD and RMSD were slightly larger for larger view zenith angles, particularly, in the forward scattering directions.

In contrast, TOC EVI had angular dependencies in its MD [Fig. 6(c)]. TOC EVI MD was the lowest for nadir to near-nadir view zenith angle ranges and increased with view zenith angles in both backward and forward scattering directions. This increasing trend was larger in the backward scattering direction than in the forward scattering direction, with the largest MD observed for the largest view zenith angle range [55 deg $\leq \theta_{v}<62.5 \mathrm{deg}$, or $-60 \mathrm{deg}$ in Fig. 6(c)]. TOC EVI RMSD had the same angular dependencies as its MDs, whereas TOC EVI SD did not change largely across view zenith angles [Fig. 6(c)]. TOC EVI2 MD and RMSD also had some angular dependency in that they were consistently slightly higher for the backward scattering conditions, but that dependency was not as strong as that observed in TOC EVI MD and RMSD [Fig. 6(d)]. TOC EVI2 SD did not change largely across view zenith angles and had the similar magnitude to TOC EVI SD. 

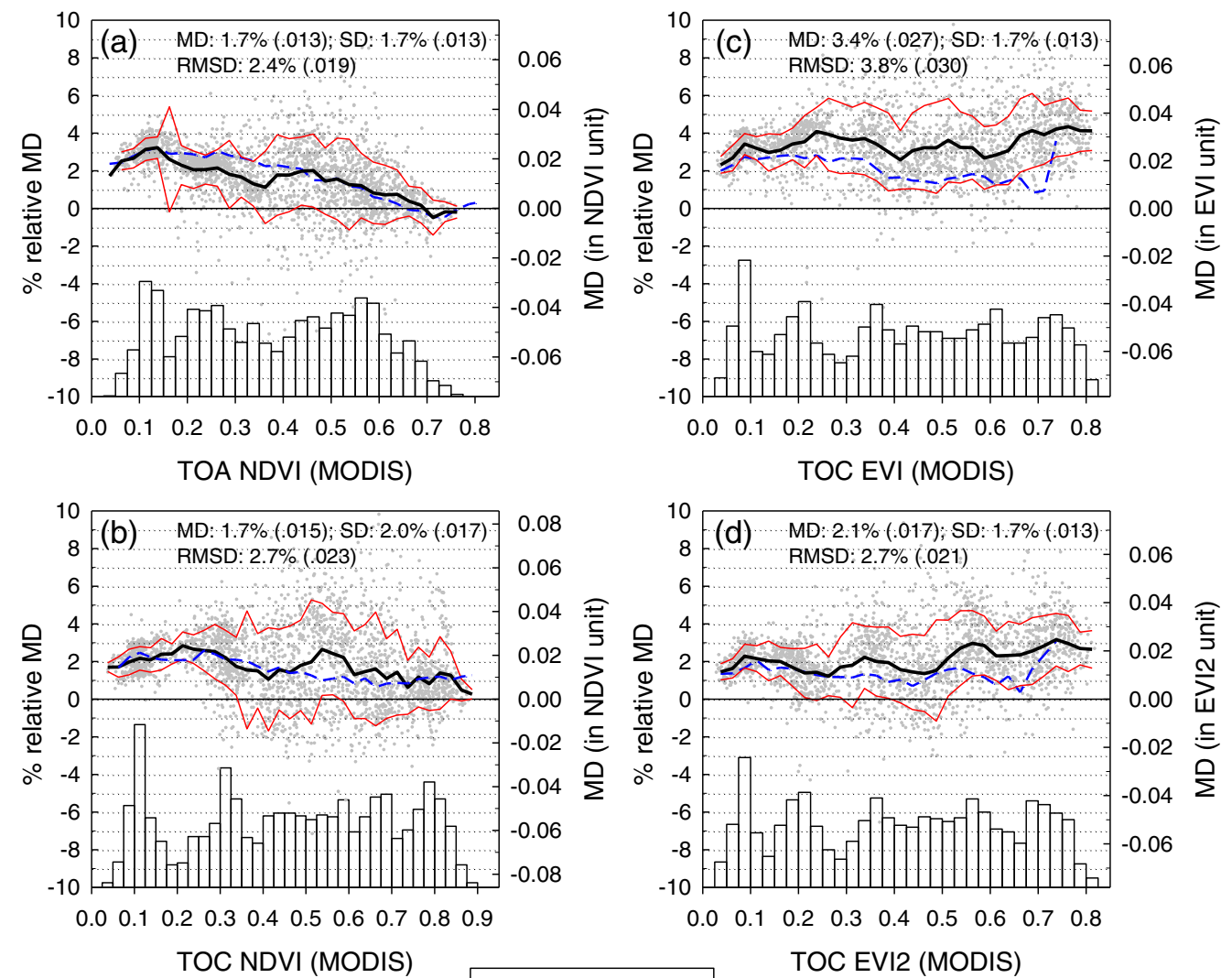

TOC NDVI (MODIS)

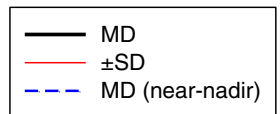

Fig. 5 Same as Fig. 4, but for off-nadir viewing in the backward scattering direction (55 deg $\leq \theta_{v}<62.5 \mathrm{deg}$ and $0 \mathrm{deg} \leq \phi_{r} \leq 90 \mathrm{deg}$ ).

\subsection{Compatibility across Seasons}

TOA NDVI MD remained nearly the same across seasons for all the view zenith angle bins [Fig. 7(a)]. Although higher or lower MDs were occasionally observed, they were not associated with any specific months of year. TOC NDVI MD varied across seasons for all the view zenith angle bins [Fig. 7(b)]. They varied slightly $(\sim 1 \%)$ for the nadir-to-near-nadir view zenith angle bins $\left(-7.5 \mathrm{deg}<\theta_{v}<7.5 \mathrm{deg}\right)$, but varied more largely $(\sim 1.5 \%)$ for larger view zenith angle bins. TOC NDVI MD was consistently lower in the April to June period (DOY 90 to 160) and the largest MDs occurred in either September ( DOY 250) or October ( DOY 280), except for the 55-deg to 62.5-deg view zenith angle bin.

TOC EVI MD varied slightly across seasons, but remained at or above 2\% [Fig. 7(c)]. TOC EVI MD had seasonal variations for the backward scattering observation geometries for which MDs were higher for the January to February and July to August periods, corresponding to the summer months in the southern and northern hemispheres, respectively. TOC EVI2 MD also varied across seasons for the off-nadir viewing geometries [Fig. 7(d)]. For the off-nadir viewing conditions, TOC EVI2 MDs were higher during the summer months (January to February and July to August) and these seasonal variations were larger for larger view zenith angle bins in the backward scattering direction [Fig. 7(d)].

In Figs. 7(e)-7(1), SDs and RMSDs of the four VIs are plotted in time series for all the view zenith angle bins. SDs seasonally varied a little for TOA NDVI, TOC EVI, and TOC EVI2 [Figs. 7(e), 7(g), and 7(h), respectively]. TOC NDVI SDs had a slightly-increasing trend for a September-December period [Fig. 7(f)]. Seasonal trends and variations in RMSDs were similar to, but less than those observed in their corresponding MD time series for all the VIs [Figs. 7(i)-7(1)]. 
(a) TOA NDVI

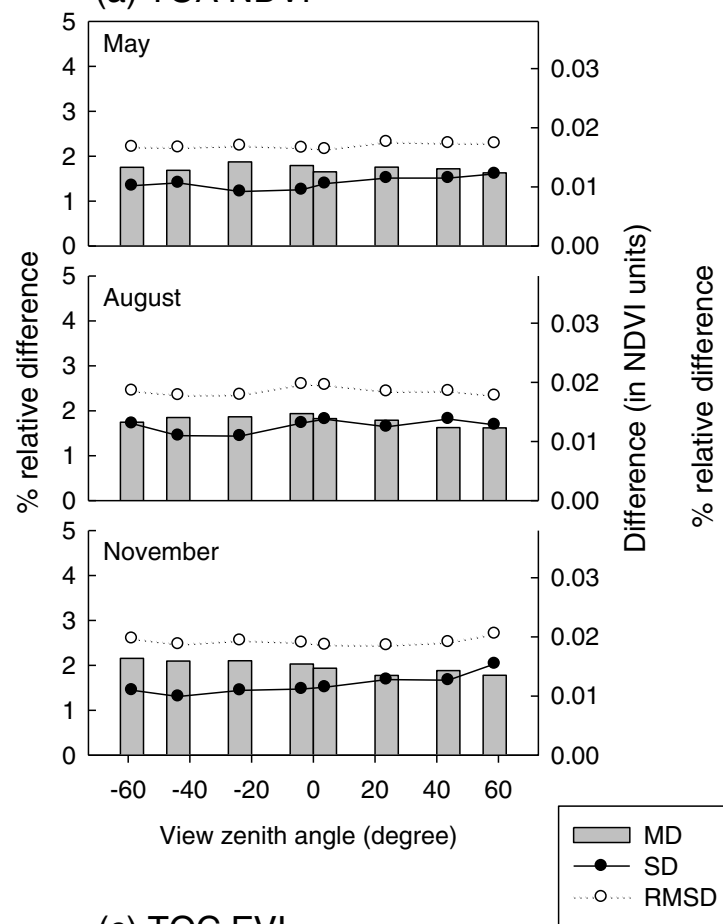

(c) TOC EVI

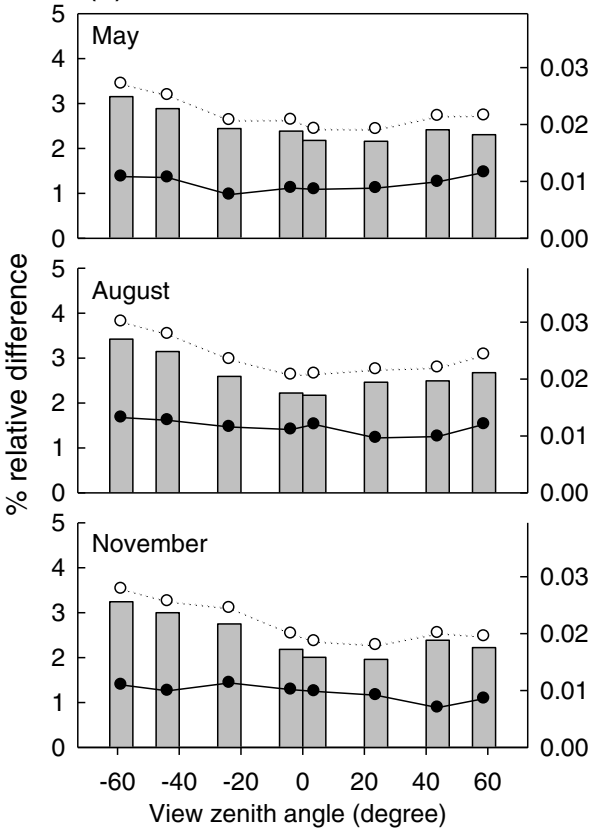

(b) TOC NDVI
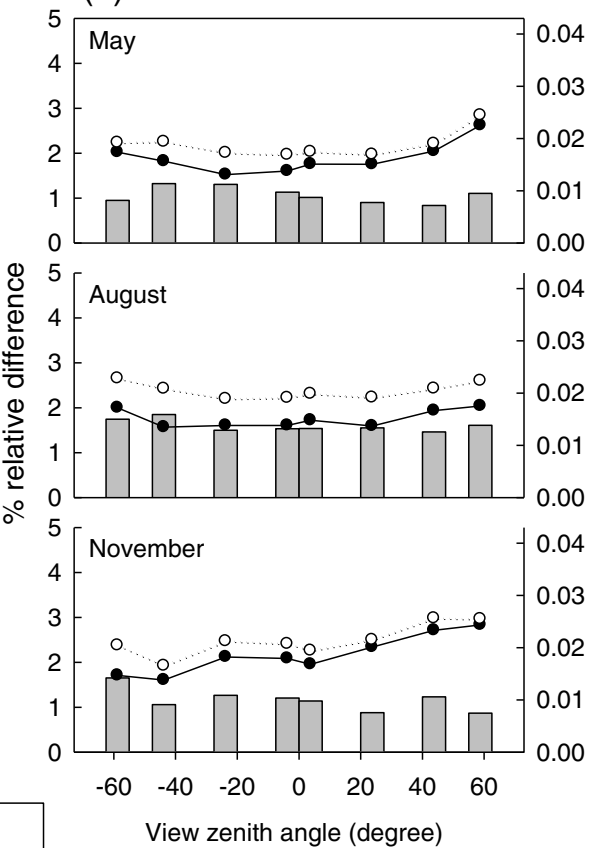

(d) TOC EVI2

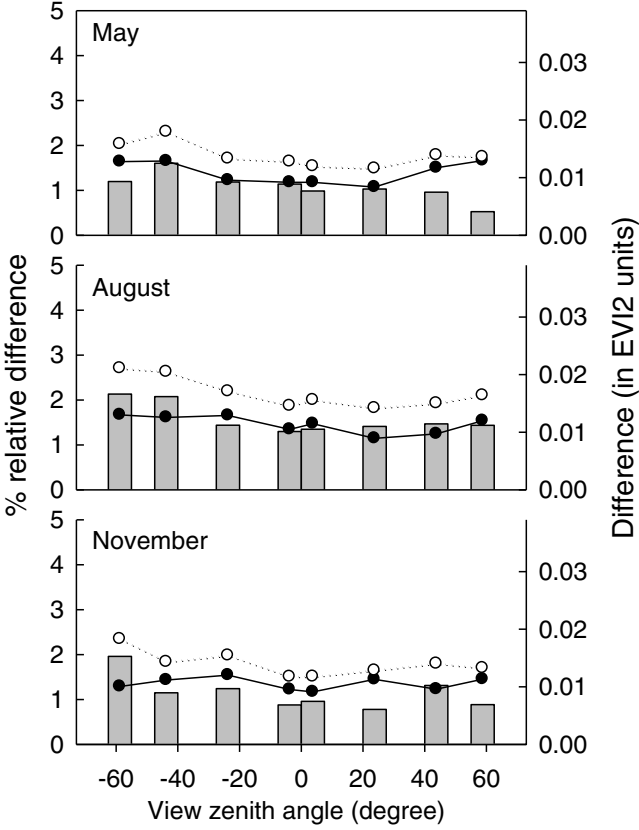

Fig. 6 Difference statistics (VIIRS minus MODIS) plotted as a function of view zenith angle for May (top), August (middle), and November (bottom): (a) TOA NDVI, (b) TOC NDVI, (c) TOC $\mathrm{EVI}$, and (d) TOC EVI2. The negative view zenith angles indicate backward scattering conditions (0 deg $\leq \phi_{r} \leq 90$ deg).

The mean MDs, SDs, and RMSDs of the four VIs are plotted in Fig. 8 to compare their overall magnitudes. The 2.5 th and 97.5 th percentiles of these difference statistics are also plotted to provide their value ranges (95\%). These mean and percentile values were obtained from the 88 values computed per month per geoangle bin (for 11 months and eight geoangle bins per month). TOC NDVI and TOC EVI2 had the smallest overall MD (below 1.5\%), whereas TOC EVI had the largest overall MD at 2.5\% (Fig. 8). However, TOC NDVI was subject to the largest mean 

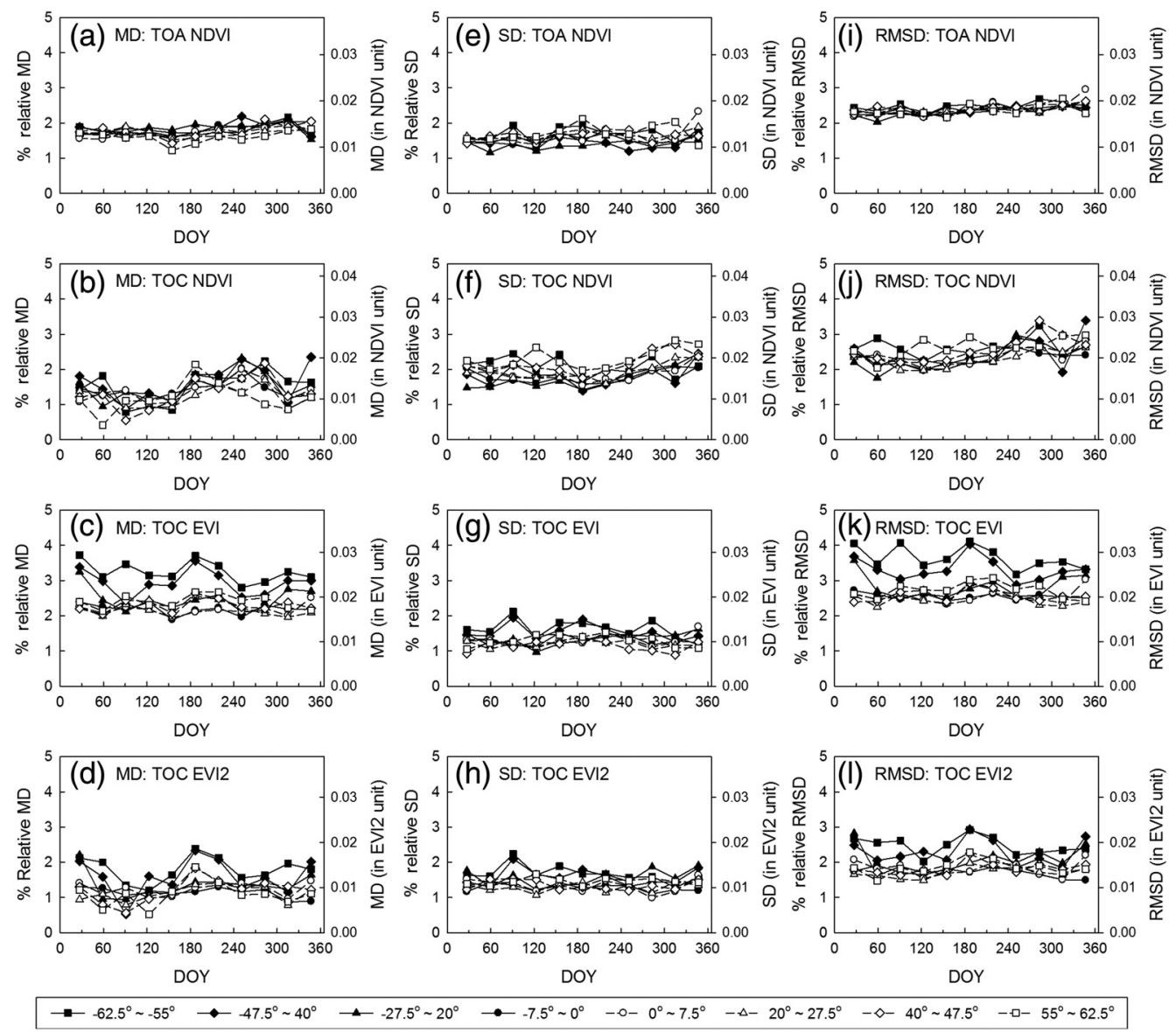

Fig. 7 Time-series plots of difference statistics (VIIRS minus MODIS) for various view zenith angles: MD, standard deviation of difference (SD), and RMSD. The negative view zenith angles indicate backward scattering conditions $\left(0 \mathrm{deg} \leq \phi_{r} \leq 90 \mathrm{deg}\right)$.

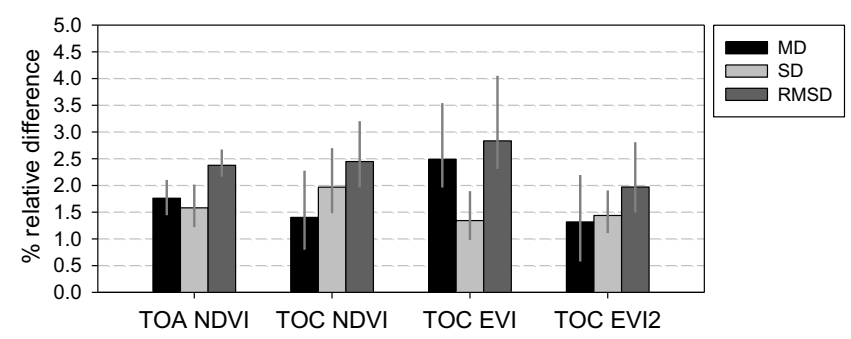

Fig. 8 Overall difference statistics between VIIRS and MODIS VIs.

Table 4 VIIRS versus MODIS VI overall difference statistics (VIIRS minus MODIS).

\begin{tabular}{lccc}
\hline \hline & MD & SD & RMSD \\
\hline TOA NDVI & 0.013 & 0.012 & 0.018 \\
TOC NDVI & 0.012 & 0.017 & 0.021 \\
TOC EVI & 0.020 & 0.011 & 0.022 \\
TOC EVI2 & 0.010 & 0.011 & 0.015 \\
\hline \hline
\end{tabular}


SD, TOA NDVI and TOC NDVI resulting in having the same level of mean RMSD (uncertainty) at $\sim 2.4 \%$. The smallest mean uncertainty was found for TOC EVI2 $(\sim 2 \%)$, whereas the largest was obtained for TOC EVI ( 2.8\%). These overall difference statistics (mean values) are provided in actual VI units in Table 4.

\subsection{Band Decomposition Analysis}

The analysis results presented in the above sections showed that the degree of VIIRS-MODIS compatibility was view zenith angle dependent for TOC EVI and, to a lesser extent, for TOC EVI2, but not for TOA NDVI and TOC NDVI. In this section, we decompose the observed VI differences into their band components to identify the spectral band(s) responsible for the observed view zenith angle dependencies of cross-sensor VI differences using the August 2015 dataset.

For the TOA NDVI, the NIR band component was the main source of VIIRS-MODIS crosssensor VI differences [Fig. 9(a)]. The NIR band component varied slightly with view zenith angles, but the red band component counter-acted for the overall NDVI differences to remain unchanged across the view zenith angles. For the TOC NDVI, both the NIR and red band components contributed approximately equally to the total NDVI differences [Fig. 9(b)].

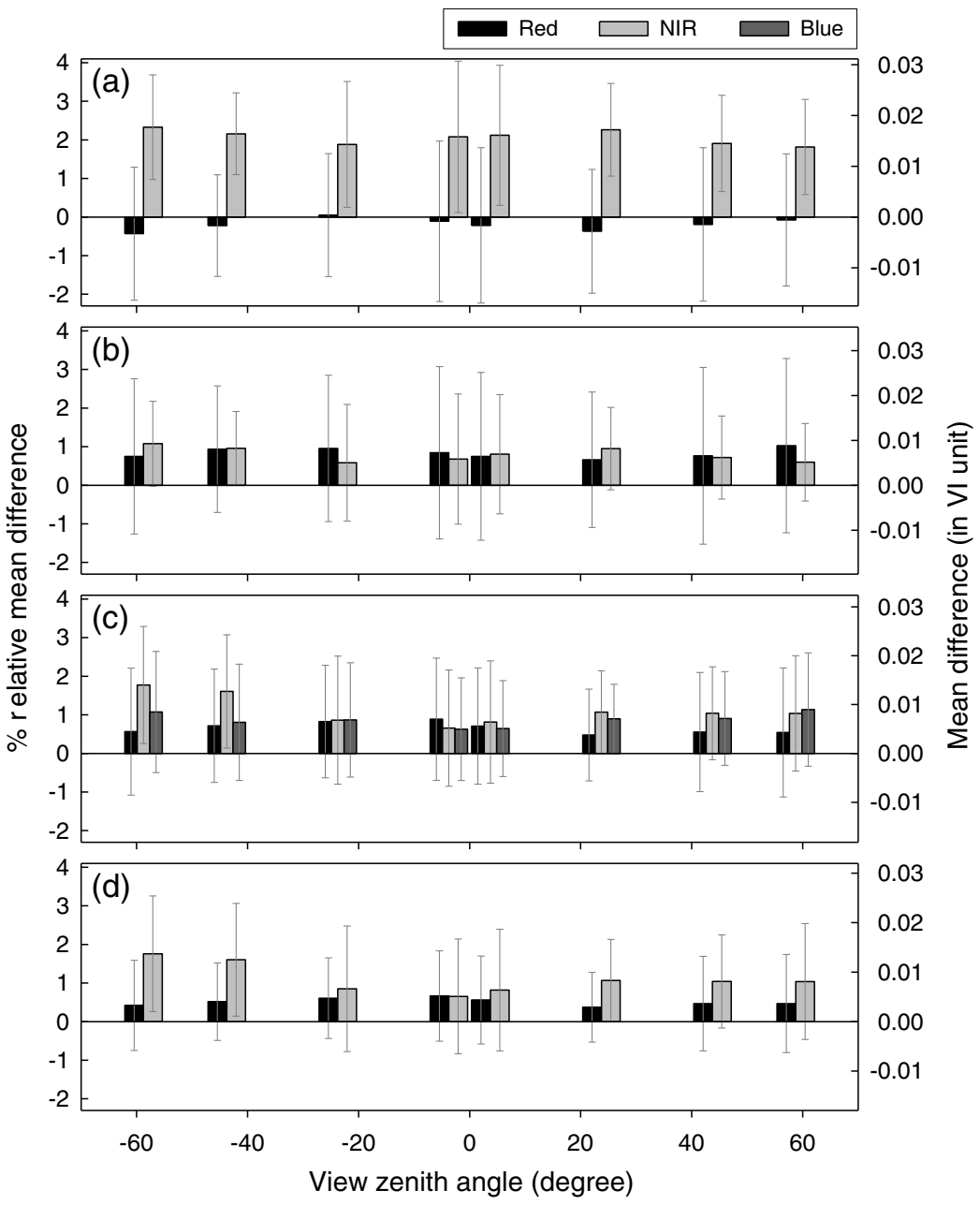

Fig. 9 VI band component differences for August 2015 (a) TOA NDVI, (b) TOC NDVI, (c) TOC $\mathrm{EVI}$, and (d) TOC EVI2. The bars and error bars correspond to the means and standard deviations of VI band component differences. The negative view zenith angles indicate backward scattering conditions ( $\left.0 \mathrm{deg} \leq \phi_{r} \leq 90 \mathrm{deg}\right)$. 
Their magnitudes were slightly different across view zenith angles, but they also counteracted for the overall NDVI differences to vary little across view zenith angles. For both the TOA and TOC NDVIs, no apparent trends were seen in the relative contributions of the red versus NIR band components with respect to view zenith angles.

In the TOC EVI, the three band components systematically varied with view zenith angles [Fig. 9(c)]. Whereas the red band component was smaller for larger view zenith angle bins, the NIR and blue band components were larger for larger view zenith angle bins [Fig. 9(c)]. Among the three band components, the NIR band component had the largest changes with view zenith angles and the largest contribution to the overall EVI differences. Thus, the NIR reflectance difference was the most responsible for the observed view zenith angle dependencies in the TOC EVI differences. In the TOC EVI2, while both the NIR and red band components also varied systematically, it is also the NIR band component that had the largest change and contribution to the overall EVI2 differences [Fig. 9(d)].

\section{Discussion}

VIIRS versus MODIS cross-sensor VI differences reported in this study were much smaller than those found between MODIS and AVHRR whose spectral bandpasses were more different than those between VIIRS and MODIS. Trishchenko et al. ${ }^{36}$ obtained the MD of 0.15 for TOA NDVI between Terra MODIS and NOAA-14 AVHRR/2 based on their temporally coincident image pairs over an Northern Ontario area in Canada. Gallo et al. ${ }^{37}$ compared Terra MODIS TOC NDVI and NOAA-16 AVHRR/3 NDVI (atmospherically corrected for the molecular scattering and gaseous absorption effects) 16-day composited time-series data for the year 2001 over the conterminous USA. The derived simple linear model (MODIS versus AVHRR) had the intercept and slope values of 0.031 and 1.009, respectively, indicating that the MD of MODIS NDVI and AVHRR NDVI was 0.031 NDVI units or higher. As the AVHRR sensor series does not include a blue band, Zhang et al. ${ }^{28}$ compared the EVI2 data between NOAA AVHRR/3 and VIIRS, and between Terra MODIS and VIIRS at the climate modeling grid resolution $(0.05 \mathrm{deg})$ for a 3-year period from 1 July 2012 to 31 June 2015. Their two-way comparative analysis results indicated that both VIIRS and MODIS EVI2 were systematically larger than the AVHRR counterpart with a larger magnitude than the VIIRS versus MODIS EVI2 difference.

The result of the present study, that inter-sensor differences in the VI anisotropy were basically significant only for the EVI, was consistent with previous studies that reported the EVI being more anisotropic than the NDVI. ${ }^{38,39}$ The same result also supports the standardization/ normalization of sun-target-viewing geometry as a required processing step for the combined uses of VIIRS and MODIS VI products. One potential standardization approach is to use observations acquired at a certain view zenith angle range where VIIRS versus MODIS EVI differences showed little view zenith angle dependency (e.g., $-40 \mathrm{deg}<\theta_{V}<40 \mathrm{deg}$ ) in generating VI time-series data. Although this would potentially reduce the temporal resolution of the derived VI time-series data, this approach would improve the consistency of the derived VI timeseries data by reducing the pixel-size variations as those deformed pixels associated with large view zenith angles, in particular for MODIS, are removed (Table 1). This "view angle constraint" approach has been one of the standard processing steps adopted in the generation of AVHRR NDVI time-series data ${ }^{40}$ and "eMODIS" products" to improve the pixel-size consistency of the products.

Another standardization approach is to normalize VI time-series data to a fixed nadir viewing and illumination geometry before the cross-sensor spectral bandpass adjustment. ${ }^{42}$ This BRDFadjustment approach would be a more desirable approach than the view angle constraint approach, as the former not only allows for the normalization of view zenith angle, but also would allow for the normalization of the VIIRS versus MODIS orbital cycle difference. Toté et al. ${ }^{43}$ also recommended the BRDF-adjustment approach to possibly adjust cross-sensor NDVI differences between Satellite Pour l'Observation de la Terre (SPOT) VEGETATION-1 and VEGETATION-2 caused by their platform orbital drift. This approach has been adopted to the Landsat- 8 and Sentinel-2 Harmonization project, where Landsat- 8 and Sentinel-2 surface 
reflectance data are normalized to nadir viewing and fixed illumination before the inter-sensor spectral bandpass adjustment, given the differing sun and view zenith angles associated with these sensor data. ${ }^{44}$

We used a stratified random sampling scheme where the three parameters of season (month), VI value, and view zenith angle were used in the stratification. Land cover type was not included as a factor in the stratification as it was practically difficult to obtain sufficient samples with the four parameters. We expect that VIIRS versus MODIS cross-sensor VI differences would likely follow the trends shown in Figs. 4-7, regardless of land cover types. Unlike some satellite biophysical products, such as LAI and GPP, no land cover dependency was assumed in deriving these VI products. There might be some geographic and/or target brightness dependencies in cross-sensor differences of TOC VIs as the accuracy of the atmospheric aerosol retrievals depends partly on these two factors.

\section{Conclusion}

In this study, we investigated cross-sensor compatibilities of the four VIs between VIIRS and MODIS, namely TOA NDVI, TOC NDVI, TOC EVI, and TOC EVI2, and the input reflectances using their near-coincident observation pairs obtained across the globe for the year 2015. All the four VIs from VIIRS and MODIS were subject to systematic differences. The systematic differences (VIIRS minus MODIS) were positive and, thus, VIIRS VIs were higher than their MODIS counterparts. The overall mean MDs and RMSDs of the four VIs were $<2.5 \%$ and $3 \%$, respectively. VIIRS versus MODIS TOA NDVI differences were the least variable across seasons and view zenith angles with the overall MD and RMSD of $0.013(1.8 \%)$ and $0.018(2.4 \%)$, respectively. TOC EVI2 had the smallest cross-sensor difference with the overall MD and RMSD of $0.010(1.3 \%)$ and $0.016(2.0 \%)$, respectively. TOC EVI was subject to the largest cross-sensor difference with the overall MD and RMSD of 0.020 (2.5\%) and $0.023(2.9 \%)$, respectively. Cross-sensor TOC NDVI difference with the overall MD of $0.012(1.4 \%)$ was as small as that of TOC EVI2, but varied across seasons, resulting in the similar overall RMSD level of 0.021 (2.5\%) to TOA NDVI.

The systematic differences of TOC EVI as well as TOC EVI2 were view zenith angle dependent. Their differences increased with increasing view zenith angles, in particular, for the backward viewing conditions. For higher view zenith angles, their systematic differences were systematically higher during the summer seasons. The analysis of crosssensor band reflectances showed that reflectance differences of all the input bands systematically varied with view zenith angles. The band decomposition analysis allowed to analyze how these input band reflectance differences were combined into the overall VI differences. The analysis results indicated that it was the NIR band component that had the largest contribution and influence on the observed view zenith angle dependency of VIIRS-MODIS EVI differences.

Research into the effectiveness of the VIIRS-MODIS spectral bandpass adjustment methods (e.g., Ref. 26) across view zenith angles and their long-term stability is currently in progress.

\section{Appendix: Partial Derivatives of Select Vegetation Indices}

\subsection{Normalized Difference Vegetation Index}

$$
\begin{aligned}
& \frac{\partial \mathrm{NDVI}}{\partial \rho_{\mathrm{NIR}}}=\frac{2 \rho_{\mathrm{red}}}{\left(\rho_{\mathrm{NIR}}+\rho_{\mathrm{red}}\right)^{2}}, \\
& \frac{\partial \mathrm{NDVI}}{\partial \rho_{\mathrm{red}}}=\frac{-2 \rho_{\mathrm{NIR}}}{\left(\rho_{\mathrm{NIR}}+\rho_{\mathrm{red}}\right)^{2}} .
\end{aligned}
$$




\subsection{Enhanced Vegetation Index}

$$
\begin{gathered}
\frac{\partial \mathrm{EVI}}{\partial \rho_{\mathrm{NIR}}}=\frac{G\left[\left(1+C_{1}\right) \rho_{\text {red }}-C_{2} \rho_{\text {blue }}+L\right]}{\left(\rho_{\mathrm{NIR}}+C_{1} \rho_{\text {red }}-C_{2} \rho_{\text {blue }}+L\right)^{2}}, \\
\frac{\partial \mathrm{EVI}}{\partial \rho_{\text {red }}}=\frac{-G\left[\left(1+C_{1}\right) \rho_{\mathrm{NIR}}-C_{2} \rho_{\text {blue }}+L\right]}{\left(\rho_{\mathrm{NIR}}+C_{1} \rho_{\text {red }}-C_{2} \rho_{\text {blue }}+L\right)^{2}}, \\
\frac{\partial \mathrm{EVI}}{\partial \rho_{\text {blue }}}=\frac{G C_{2}\left(\rho_{\mathrm{NIR}}-\rho_{\text {red }}\right)}{\left(\rho_{\mathrm{NIR}}+C_{1} \rho_{\text {red }}-C_{2} \rho_{\text {blue }}+L\right)^{2}} .
\end{gathered}
$$

\subsection{Two-Band, Enhanced Vegetation Index}

$$
\begin{gathered}
\frac{\partial \mathrm{EVI} 2}{\partial \rho_{\mathrm{NIR}}}=\frac{2.5\left(3.4 \rho_{\mathrm{red}}+1\right)}{\left(\rho_{\mathrm{NIR}}+2.4 \rho_{\mathrm{red}}+1\right)^{2}} \\
\frac{\partial \mathrm{EVI} 2}{\partial \rho_{\mathrm{red}}}=\frac{-2.5\left(3.4 \rho_{\mathrm{NIR}}+1\right)}{\left(\rho_{\mathrm{NIR}}+2.4 \rho_{\mathrm{red}}+1\right)^{2}} .
\end{gathered}
$$

\section{Acknowledgments}

This study was supported by a NOAA JPSS contract to University of Hawaii at Manoa (T.M.). The views, opinions, and findings contained in this paper are those of the authors and should not be construed as a position, policy, or decision of the U.S. Government or the National Oceanic and Atmospheric Administration.

\section{References}

1. R. B. Myneni et al., "Increased plant growth in the northern high latitudes from 1981 to 1991," Nature 386(6626), 698-702 (1997).

2. G. E. P. Campos et al., "Ecosystem resilience despite large-scale altered hydroclimatic conditions," Nature 494(7437), 349-352 (2013).

3. X. Y. Zhang, M. A. Friedl, and C. B. Schaaf, "Global vegetation phenology from moderate resolution imaging spectroradiometer (MODIS): evaluation of global patterns and comparison with in situ measurements," J. Geophys. Res. 111(G4), G04017 (2006).

4. R. R. Nemani et al., "Climate-driven increases in global terrestrial net primary production from 1982 to 1999," Science 300(5625), 1560-1563 (2003).

5. L. Jiang et al., "Real-time weekly global green vegetation fraction derived from advanced very high resolution radiometer-based NOAA operational global vegetation index (GVI) system," J. Geophys. Res. 115(D11), D11114 (2010).

6. K. Ichii et al., "New data-driven estimation of terrestrial $\mathrm{CO} 2$ fluxes in Asia using a standardized database of eddy covariance measurements, remote sensing data, and support vector regression," J. Geophys. Res. 122(4), 767-795 (2017).

7. J. W. Rouse et al., "Monitoring vegetation systems in the Great Plains with ERTS," in Third Earth Resources Technology Satellite-1 Symp., Vol. 1, NASA SP-351, pp. 309-317 (1973).

8. C. J. Tucker, "Red and photographic infrared linear combinations for monitoring vegetation," Remote Sens. Environ. 8(2), 127-150 (1979).

9. E. J. Pinzon and J. C. Tucker, "A non-stationary 1981-2012 AVHRR NDVI3g time series," Remote Sens. 6(8), 6929-6960 (2014).

10. B. Chen et al., "Changes in vegetation photosynthetic activity trends across the Asia-Pacific region over the last three decades," Remote Sens. Environ. 144, $28-41$ (2014).

11. A. Huete et al., "Overview of the radiometric and biophysical performance of the MODIS vegetation indices," Remote Sens. Environ. 83(1-2), 195-213 (2002). 
Miura, Muratsuchi, and Vargas: Assessment of cross-sensor vegetation index compatibility...

12. S. R. Saleska et al., "Dry-season greening of Amazon forests," Nature 531(7594), E4-E5 (2016).

13. A. W. R. Seddon et al., "Sensitivity of global terrestrial ecosystems to climate variability," Nature 531(7593), 229-232 (2016).

14. A. F. Rahman et al., "Potential of MODIS EVI and surface temperature for directly estimating per-pixel ecosystem C fluxes," Geophys. Res. Lett. 32(19), L19404 (2005).

15. M. Vargas et al., "An initial assessment of Suomi NPP VIIRS vegetation index EDR," J. Geophys. Res. 118(22), 1-16 (2013).

16. Z. Jiang et al., "Development of a two-band enhanced vegetation index without a blue band," Remote Sens. Environ. 112(10), 3833-3845 (2008).

17. K. Didan et al., "Multi-sensor vegetation index and phenology earth science data records: algorithm theoretical basis document and user guide," Version 4.0 (2015).

18. Y. Kim et al., "Response of vegetation growth and productivity to spring climate indicators in the conterminous United States derived from satellite remote sensing data fusion," Agric. For. Meteorol. 194, 132-143 (2014).

19. X. Zhang, B. Tan, and Y. Yu, "Interannual variations and trends in global land surface phenology derived from enhanced vegetation index during 1982-2010," Int. J. Biometeorol. 58(4), 547-564 (2014).

20. J. Liu, E. Pattey, and G. Jego, "Assessment of vegetation indices for regional crop green LAI estimation from Landsat images over multiple growing seasons," Remote Sens. Environ. 123, 347-358 (2012).

21. C. Cao et al., "Suomi NPP VIIRS sensor data record verification, validation, and long-term performance monitoring," J. Geophys. Res. 118(20), 11,664-11,678 (2013).

22. X. Xiong et al., "VIIRS on-orbit calibration methodology and performance," J. Geophys. Res. 119(9), 5065-5078 (2014).

23. Y. Kim et al., "Spectral compatibility of vegetation indices across sensors: a band decomposition analysis with Hyperion data," J. Appl. Remote Sens. 4, 043520 (2010).

24. T. Miura, J. P. Turner, and A. R. Huete, "Spectral compatibility of the NDVI across VIIRS, MODIS, and AVHRR: an analysis of atmospheric effects using EO-1 Hyperion," IEEE Trans. Geosci. Remote Sens. 51(3), 1349-1359 (2013).

25. T. Miura et al., "Inter- and intra-sensor spectral compatibility and calibration of the enhanced vegetation indices," in Remotely Sensed Data Characterization, Classification, and Accuracies, P. S. Thenkabail, Ed., pp. 155-174, Taylor and Francis Inc.ICRC Press, Boca Raton, Florida (2015).

26. K. Obata et al., "Spectral cross-calibration of VIIRS enhanced vegetation index with MODIS: a case study using year-long global data," Remote Sens. 8(1), 34 (2016).

27. S. Skakun et al., "Transitioning from MODIS to VIIRS: an analysis of inter-consistency of NDVI data sets for agricultural monitoring," Int. J. Remote Sens. 39(4), 971-992 (2018).

28. X. Zhang, L. Liu, and D. Yan, "Comparisons of global land surface seasonality and phenology derived from AVHRR, MODIS, and VIIRS data," J. Geophys. Res. 122(6), 1506-1525 (2017).

29. R. E. Wolfe et al., "Suomi NPP VIIRS prelaunch and on-orbit geometric calibration and characterization," J. Geophys. Res. 118(20), 11,508-11,521 (2013).

30. NASA Goddard Space Flight Center, "Level-1 and atmospheric archive \& distribution system distributed active archive center, 2018, https://ladsweb.modaps.eosdis.nasa.gov (18 September 2018).

31. STAR Joint Polar Satellite System Website, "JPSS/SNPP algorithm maturity matrix," 2018, https://www.star.nesdis.noaa.gov/jpss/AlgorithmMaturity.php (21 August 2018).

32. U.S. Department of Commerce, "Joint polar satellite system program level 1 requirements supplement," Version 2.10 (2014).

33. NASA Goddard Space Flight Center, "MODIS land team validation," 2018, https://landval .gsfc.nasa.gov (03 August 2018).

34. E. Swinnen and F. Veroustraete, "Extending the SPOT-VEGETATION NDVI time series (1998-2006) back in time with NOAA-AVHRR data (1985-1998) for Southern Africa," IEEE Trans. Geosci. Remote Sens. 46(2), 558-572 (2008). 
35. P. R. Bevington and D. K. Robinson, Data Reduction and Error Analysis for the Physical Sciences, McGraw-Hill, Boston (2003).

36. A. P. Trishchenko, J. Cihlar, and Z. Li, "Effects of spectral response function on surface reflectance and NDVI measured with moderate resolution satellite sensors," Remote Sens. Environ. 81(1), 1-18 (2002).

37. K. Gallo et al., "Comparison of MODIS and AVHRR 16-day normalized difference vegetation index composite data," Geophys. Res. Lett. 31(7), L07502 (2004).

38. Y. M. Moura et al., "Use of MISR/Terra data to study intra- and inter-annual EVI variations in the dry season of tropical forest," Remote Sens. Environ. 127, 260-270 (2012).

39. D. A. Sims et al., "Seasonal and inter-annual variation in view angle effects on MODIS vegetation indices at three forest sites," Remote Sens. Environ. 115(12), 3112-3120 (2011).

40. C. J. Tucker et al., "An extended AVHRR 8-km NDVI dataset compatible with MODIS and SPOT vegetation NDVI data," Int. J. Remote Sens. 26(20), 4485-4498 (2005).

41. C. Jenkerson, T. K. Maiersperger, and G. Schmidt, "eMODIS-a user-friendly data source," U.S. Geological Survey Open-File Report 2010-1055, p. 28 (2010).

42. Y. Liu et al., "Evaluation of the VIIRS BRDF, Albedo and NBAR products suite and an assessment of continuity with the long term MODIS record," Remote Sens. Environ. 201, 256-274 (2017).

43. C. Toté et al., "Evaluation of the SPOT/VEGETATION collection 3 reprocessed dataset: surface reflectances and NDVI," Remote Sens. Environ. 201, 219-233 (2017).

44. M. Claverie et al., "Harmonized landsat-8 sentinel-2 (HLS) product user's guide," http://hls .gsfc.nasa.gov (2017).

Tomoaki Miura is a professor of remote sensing and geospatial analysis at the University of Hawaii at Manoa. His research interests include multisatellite/-sensor data continuity and vegetation index product development. He is an active member of the Joint Polar Satellite System Science Team, focusing on the validation of Visible Infrared Imaging Radiometer Suite (VIIRS) Vegetation Index products, and co-leads the Vegetation Index focus area within the Committee on Earth Observation Satellites (CEOS) Working Group on Calibration/Validation (WGCV) Land Product Validation (LPV) subgroup.

Jordan Muratuchi recently graduated with his master's in natural resources and environmental management at University of Hawaii, Manoa, where he studied geospatial and remote sensing analyses, focusing his personal work on marine debris. Currently, he is the executive director of Keep the Hawaiian Islands Beautiful, a Hawaii-based nonprofit focused on promoting environmental stewardship and positive social behavior change. Additionally, he is the Hawaii state leader in the Keep America Beautiful system.

Marco Vargas received his $\mathrm{PhD}$ in electrical engineering specializing in remote sensing from the City University of New York in 2007. He was a research scientist at NOAA STAR for 2007 to 2017 where he served as the algorithm lead of the JPSS VIIRS Vegetation Index (VI) Environmental Data Record (EDR) and the lead of the VIIRS Green Vegetation Fraction (GVF) project. Currently, he is part of a team developing a new operational space weather mission. 